\title{
The Arterial Supply of the Dura Mater of the Rhesus Monkey '
}

\author{
WALTER A. CASTELLI AND DONALD F. HUELKE \\ Department of Anatomy, The University of Michigan, Ann Arbor, Michigan
}

\begin{abstract}
A study of the dural blood supply in 20 specimens by dissection, corrosion preparations and cleared specimens indicates that the dural arteries are similar to those of man yet significant differences were noted. The anterior cranial fossa is supplied by small twigs which spread through the dura of the anterior cranial fossa floor. The larger branch, the anterior meningeal artery, perforates the lateral orbital wall and supplies the lateral wall of anterior and middle cranial fossae. The middle meningeal artery, of the maxillary artery, divides into two branches in the floor of the middle fossa. The anterior or frontal branch always anastomoses with the anterior meningeal artery. Sometimes it also continues upward to the lateral wall of the middle fossa to supply it. The posterior middle meningeal branch passes backward to supply the parietal area. The posterior meningeal artery, of the occipital artery, enters the skull near the beginning of the sigmoid sinus and follows the general course of the lambdoidal suture.
\end{abstract}

The arterial supply of the dura mater in the Rhesus monkey is similar to that of man, yet several characteristics are worthy of note. Because of the importance of the dural blood supply in certain experimental neurological and surgical studies, we felt that a description of the arterial supply of the dura mater in the Rhesus monkey would be of value. Our interest in this problem was initiated through studies of the anatomy of the Rhesus monkey (Schwartz and Huelke, '63; Castelli and Huelke, '65).

\section{MATERIALS AND METHODS}

In this study 20 young Rhesus monkeys, typical of those used in neurological experiments, were used. Twelve of the specimens were unembalmed. All animals were injected through the common carotid arteries; red latex, Teichmann's paste (Teichmann, '52), or a modified Schlessinger's radiopaque media (Reiner and Rodriguez, '57) was used.

The 12 unembalmed specimens were injected with Teichmann's paste and with the radiopaque material. The heads were cut sagittally and fixed in $10 \%$ formalin solution. The brains of those injected with the radiopaque media and the soft tissues covering the skull were removed. The temporalis muscle was detached from its origin and cut transversely at the level of the zygomatic arch. In this way all the superficial arteries were removed and only those arteries of the dura mater would appear in the radiographs. The calvariae of the specimens injected with Teichmann's paste were separated from the facial structures and all soft tissues removed from the calvariae except for the dura mater. The specimens were then decalcified and cleared (Teichmann, '52).

The embalmed material was injected with red latex, the heads cut sagittally, and the dura mater carefully dissected in order to determine the vascular pathways of the meningeal arteries. All arteries were followed from their point of origin. Sketches of the arterial patterns were made, and the pathways of the vessels were illustrated with reference to certain cranial landmarks.

\section{RESULTS AND DISCUSSION}

Anterior meningeal artery. In the Rhesus monkey the anterior meningeal artery arises from the ophthalmic artery within the orbit and perforates the orbit at the junction of the superior and lateral orbital walls; it then follows the curvature of the lesser wing of the sphenoid (fig. 1). Commonly this vessel is very large and was

\footnotetext{
1 This investigation was supported, in part, by USPHS research grant DE-00895 from the National Institute of Dental Research, National Institutes of Health.
} 


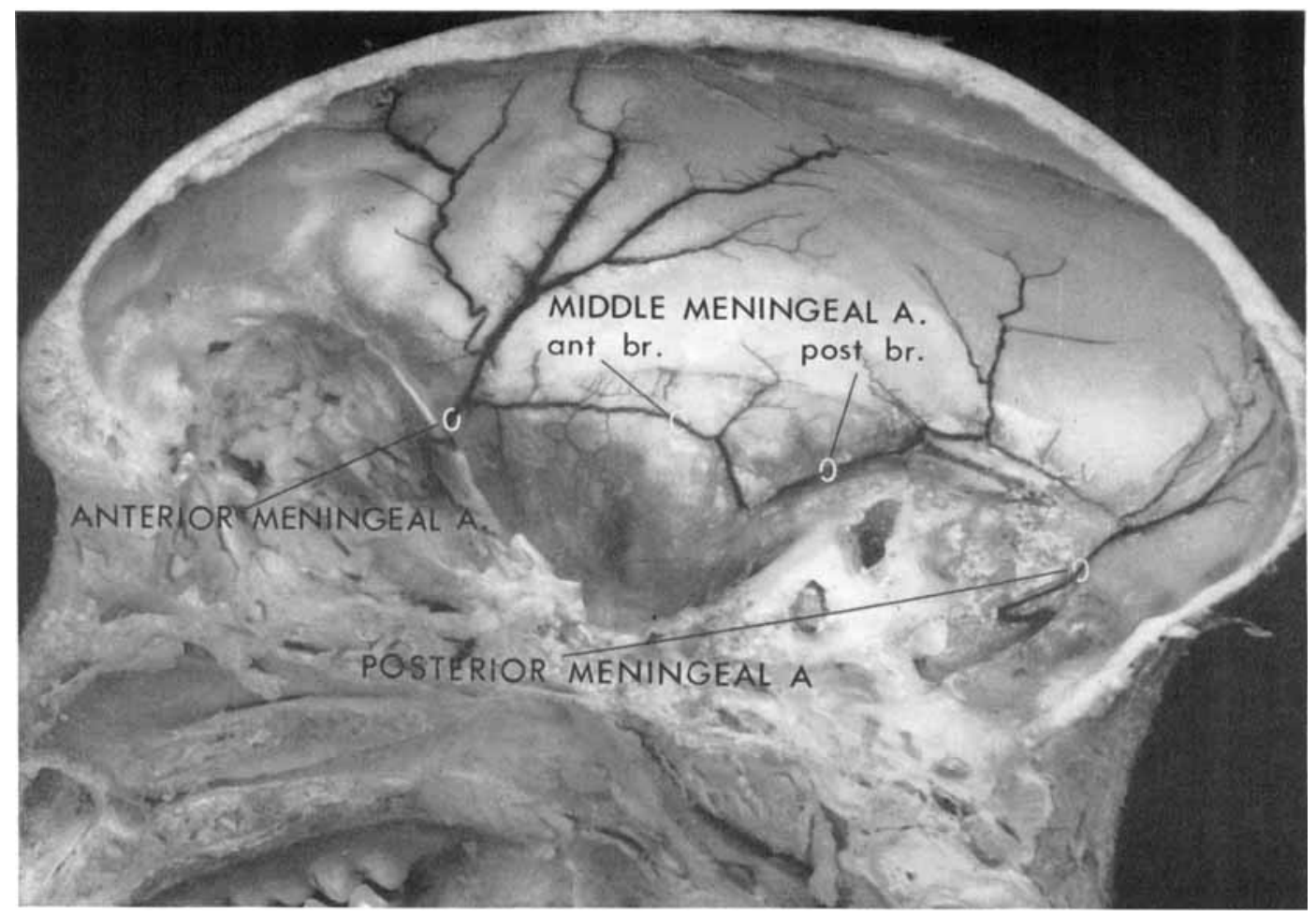

Fig. 1 The dural arteries of the right side as found in $52 \%$ of the sides. The anterior branch of the middle meningeal artery is reduced to only a small anastomotic connection which joins the anterior meningeal artery of the ophthalmic artery.

present in $80 \%$ of the material. The artery anastomoses with the frontal or anterior branch of the middle meningeal artery and then continues by dividing into two or three smaller branches which distribute to the lateral wall of the anterior and middle cranial fossae. In addition, the floor of the anterior cranial fossa is supplied by very small arterial branches which pass through foramina adjacent to the cribiform plate. These vessels arise from the internal nasal artery, a branch of the ophthalmic artery.

Middle meningeal artery. The middle meningeal artery most often arises in common with the tympanic artery. This common trunk originates from the first portion of the maxillary artery, medial to the neck of the condyle. This common trunk passes beneath the inferior border of the lateral pterygoid muscle and passes medially up- ward toward the petrotympanic fissure. Near the base of the skull the artery divides into its two main branches. The tympanic artery passes posteriorly into the petrotympanic fissure. The middle meningeal artery passes through the lateral end of the foramen ovale to enter the middle cranial fossa. It grooves the inner table of bone at the base of the middle cranial fossa immediately above the glenoid cavity as it passes toward the lateral wall. Here the artery divides into two main branches which course anteriorly and posteriorly. The anterior or frontal branch passes on the inner aspect of the squamous portion of the temporal bone towards the tip of the lesser wing of the sphenoid where it always anastomoses with the anterior meningeal branch of the ophthalmic artery when this artery is present. The posterior or parietal branch passes back- 
ward above the level of the external acoustic meatus to anastomose with the posterior meningeal artery. This anastomosis is, however, quite small. Through this branching pattern, then, the middle meningeal artery supplies the base of the middle cranial fossa and part of the lateral wall, as well as most of the area behind and above the petrous bone.

Posterior meningeal artery. The posterior meningeal artery is a branch of the occipital artery which penetrates the skull through a foramen at the occipitomastoid suture. Upon entrance into the skull it is located at the beginning of the sigmoid sinus. The artery has a tortuous course, passing backwards between the bone and the lateral dural venous sinus. It distributes to the dura mater of the posterior fossa, falx cerebelli and the tentorium cerebelli. Small branches anastomose with the parietal branch of the middle meningeal artery.

The meningeal arteries of one side anastomose with each other and with those of the opposite side by means of short transverse communications which pass across the midline above the superior sagittal sinus.

Three main patterns of arterial distribution were found. The most common vascular arrangement was noted in $52 \%$ of the specimens. Here the anterior meningeal artery is very well developed and distributed to most of the lateral wall of the anterior and middle cranial fossa. In these specimens the anterior branch of the middle meningeal artery is reduced to a very small anastomotic arch which passes forward to join the anterior meningeal artery (fig. 1). The posterior or parietal branch of the middle meningeal is much

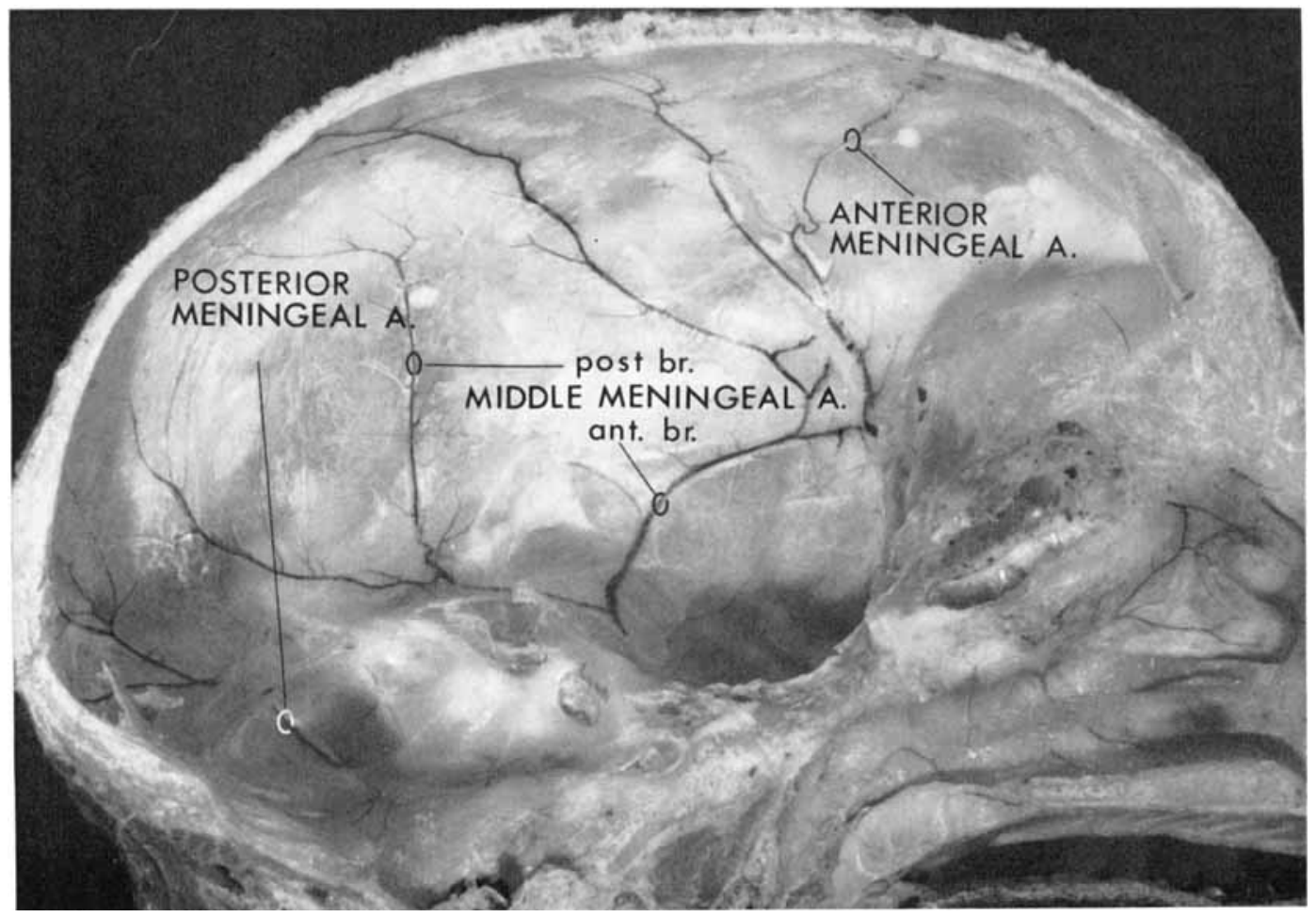

Fig. 2 Dural arteries found in $28 \%$ of sides. Anterior branch of the middle meningeal artery supplies the side of the skull above the middle cranial fossa along with the anterior meningeal artery by separate branches from the parent stem. 


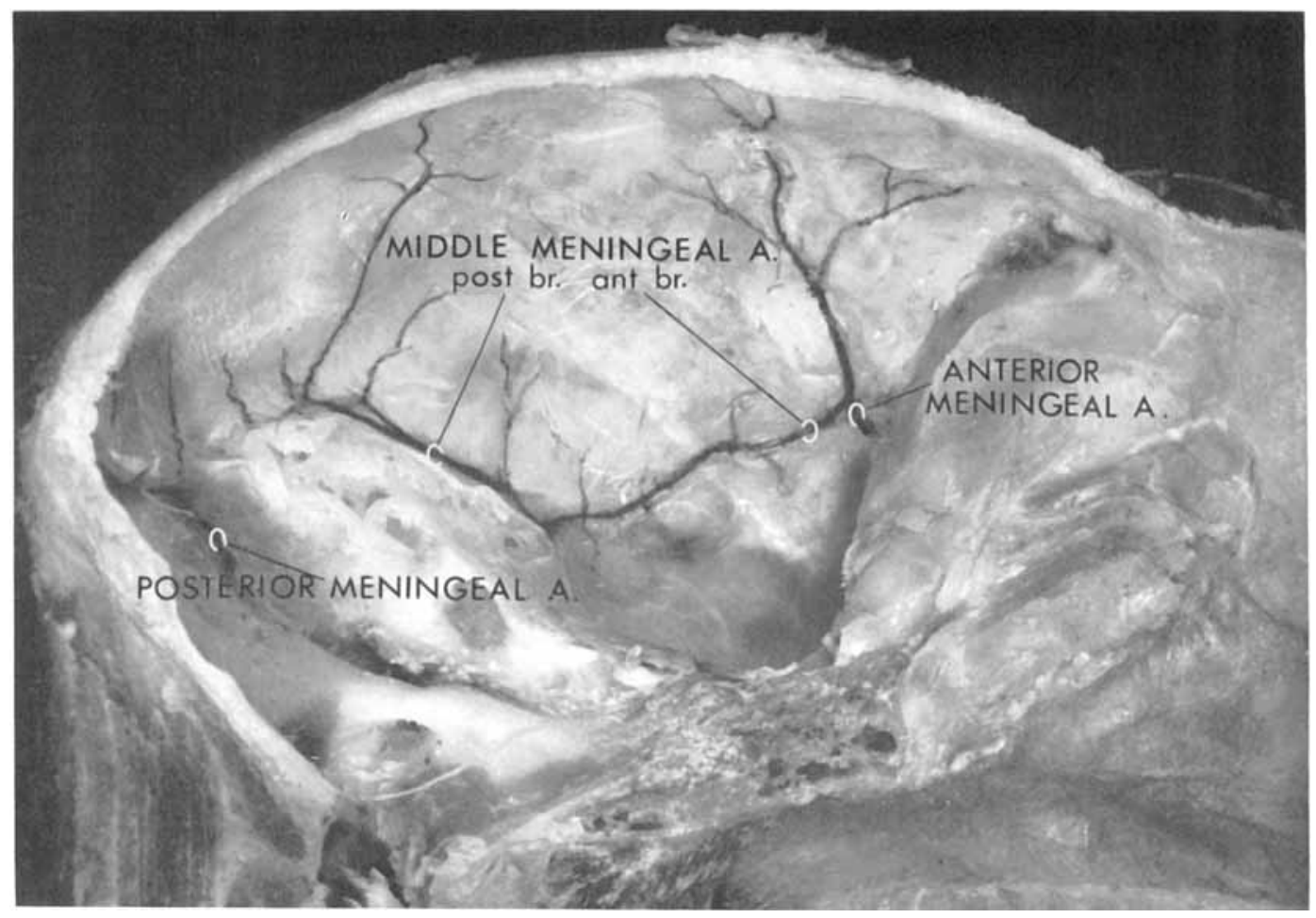

Fig. 3 Variation of the pattern seen in figure 2. The dura above the middle cranial fossa is supplied by a common stem which is formed by the anterior meningeal artery and the anterior branch of the middle meningeal artery.

larger and supplies the posterior part of the middle cranial fossa and the area behind and above the petrous temporal bone. The posterior meningeal is found in its usual location and distribution in the posterior fossa. The posterior meningeal artery is the most stable of all of the meningeal arteries in that no variations of significance were found.

The second most common vascular arrangement occurs in $28 \%$ of the sides. Here the anterior branch of the middle meningeal artery is much larger and, together with the anterior meningeal artery, participates in the supply of the dura mater of the anterior and middle cranial fossae. The vessels may remain separate, joining one another by only short anastomotic channels (fig. 2), or they may unite to form a common trunk which is then distributed to the area of the middle cranial fossa (fig.
$3)$. In these cases the posterior branch of the middle meningeal artery and the posterior meningeal artery have the usual course and distribution as previously described.

The third pattern of meningeal vasculature was seen in $20 \%$ of the specimens. Here there is a predominance of the middle meningeal artery over the others. The middle meningeal artery distributes to most of the anterior and middle cranial fossae with the anterior meningeal artery being very small or absent. Only small arterial vessels from the internal nasal artery were present in the dura mater of the floor of the anterior cranial fossa (fig. 4).

The projection of the meningeal arteries on the outer surface of the cranial vault indicates that the main meningeal trunks are located at the base of the cranium and cannot be visualized in lateral view. In profile view the anterior meningeal ar- 


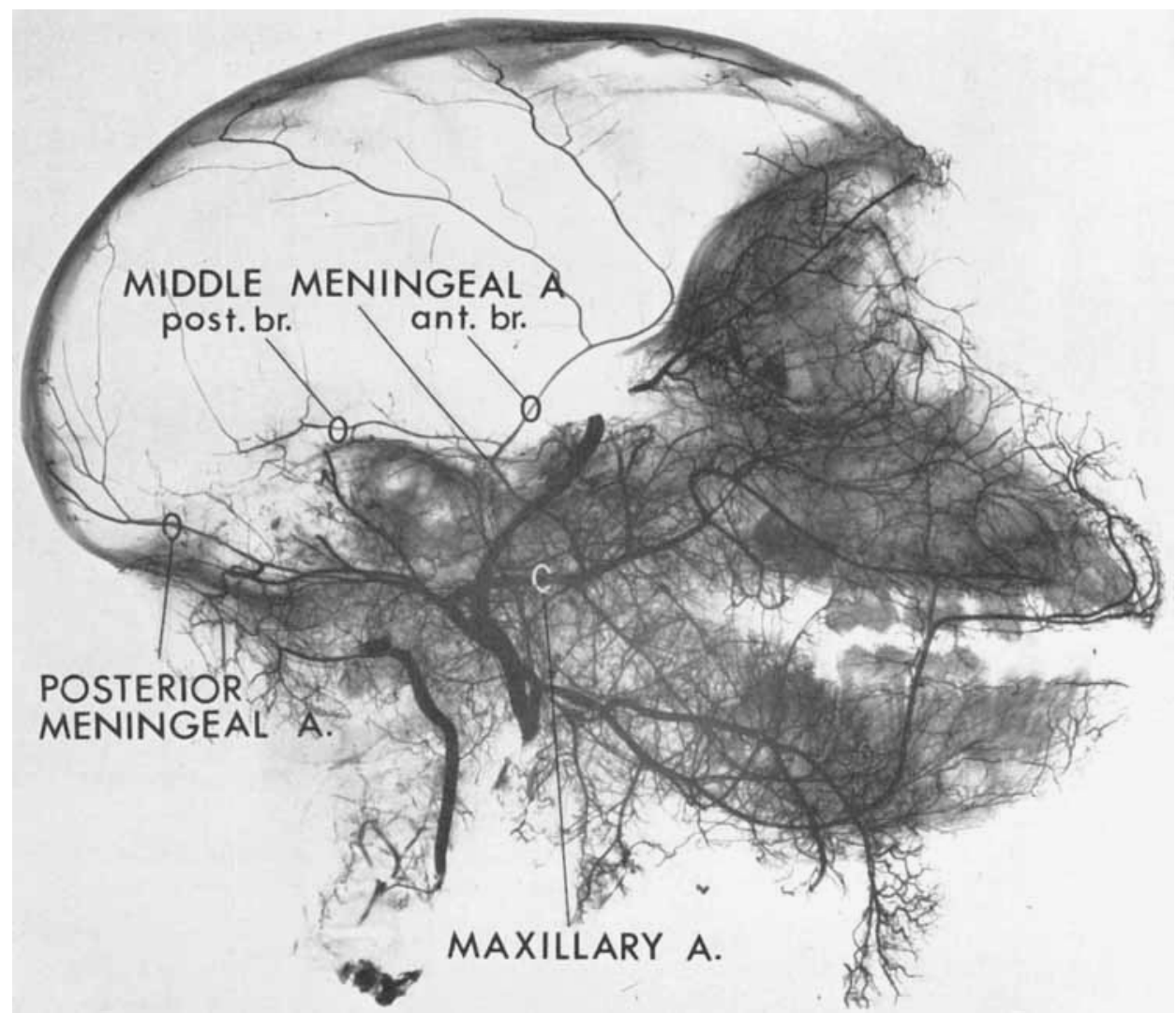

Fig. 4 Radiograph of the right side showing the distribution of the middle meningeal artery. The arterial stem on the frontal bone near the midline is the supraorbital artery.

tery is located almost at the tip of the greater wing of the sphenoid bone; the middle meningeal artery just above the glenoid fossa and external acoustic meatus; the posterior meningeal artery at the area of the junction of the lateral and sigmoid sinuses approximately $2 \mathrm{~cm}$ behind the external acoustic meatus. As indicated in figure 5 the distribution of the meningeal branches shows an area of concentration of arteries about the junction of the frontal, parietal, and sphenoid bones (pterygon). This is located approximately $\mathbf{1} \mathrm{cm}$ behind the zygomaticofrontal suture. The posterior branch of the middle meningeal artery is located in lateral view passing on a $45^{\circ}$ angle upward and backward from in front of the external acoustic meatus.
The posterior meningeal artery passes backward slightly below the lambdoidal suture.

The arrangement of the meningeal vessels also leaves areas of decreased vascular density. In these areas no major dural arterial stems or branches are found. These are located mainly near the midline, and in the lateral part of the parietal bone approximately $3 \mathrm{~cm}$ above the external acoustic meatus, and at the frontal area on either side of the midline above the orbits. In the occipital region there are but small areas just lateral to the confluens of sinuses above and below the lateral dural sinuses which correspond to the cerebral and cerebellar fossae of the occipital bone. 


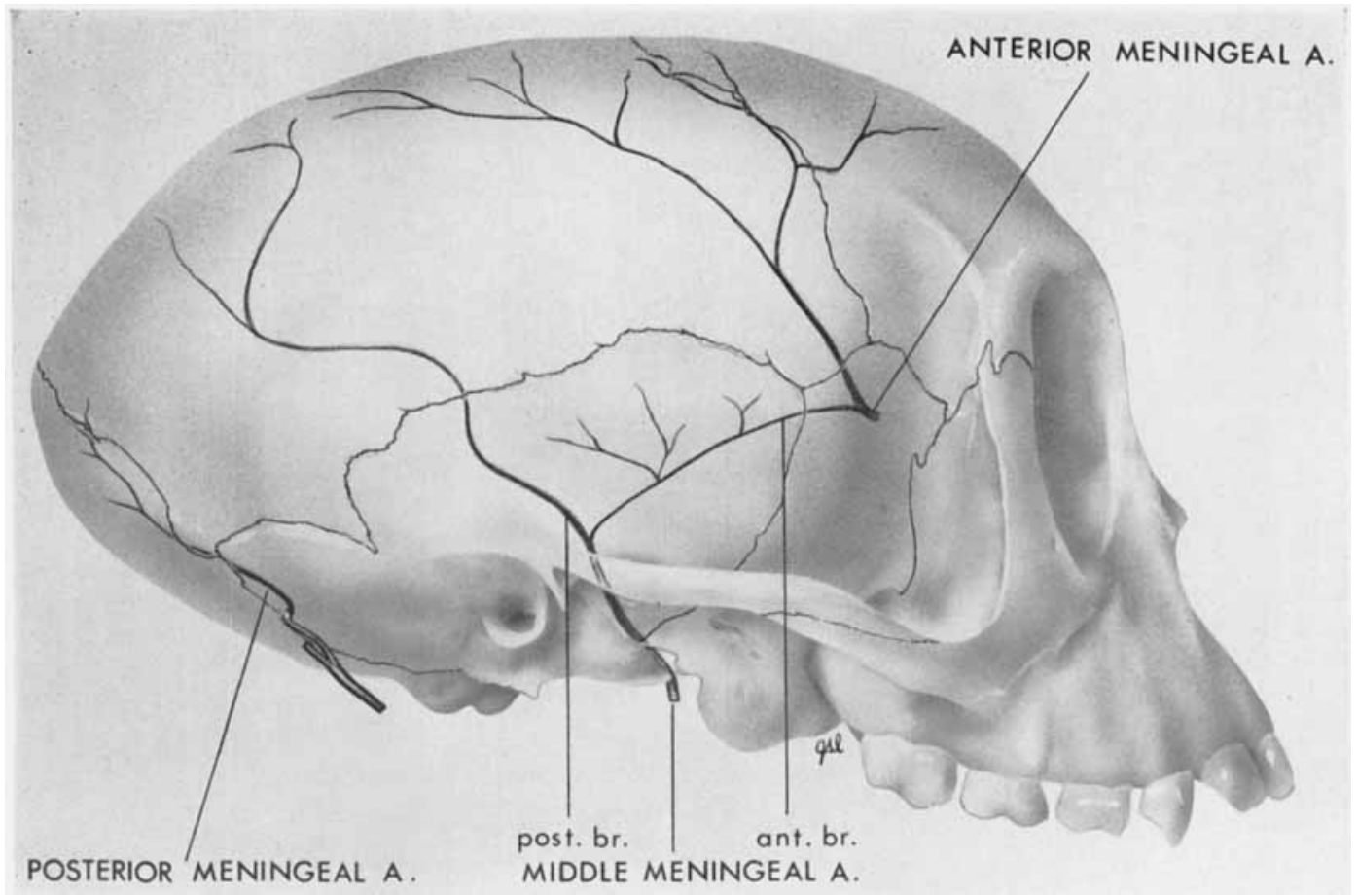

Fig. 5 The arteries of the dura mater as seen in lateral view.

\section{LITERATURE CITED}

Castelli, W. A., and D. F. Huelke 1965 The arterial system of the head and neck of the Rhesus monkey with emphasis on the external carotid system. Am. J. of Anat., 116: 149-170. Reiner, L., and F. Rodriguez 1957 An injection mass of maximal radiopacity for postmortem angiography. J. Mount Sinai Hospital, 24: 1139-1145.
Schwartz, D. J., and D. F. Huelke 1963 The morphology of the head and neck of the Macaca monkey: the muscles of mastication and the mandibular division of the trigeminal nerve. J. Dent. Res., 42: 1222-1233.

Teichmann, L. 1952 Cited by Schwering; Anatomische Trochen-feucht und Knochenpraparate. Springer Verlag, Berlin, p. 79. 